Pacific Journal of Mathematic 


\section{LIE AND JORDAN STRUCTURES IN BANACH ALGEBRAS}

\section{Paul Civin AND Bertram Yood}

We first consider the theory of Jordan homomorphisms and Jordan ideals in Banach algebras. If $B$ is a $\mathbf{B}^{*}$-algebra or a semi-simple annihilator algebra, any closed Jordan ideal in $B$ is a two-sided ideal. Any Jordan homomorphism of a Banach algebra onto $B$ is automatically continuous. That Jordan homomorphisms are continuous and Jordan ideals are ideals is shown to hold in a number of other situations. We also study the Lie ideals in a semi-simple Banach algebra $A$. If the center of $A$ is zero and proper closed Lie ideals do not contain their Lie annihilators, then $A$ is direct topological sum of its minimal closed ideals. An $H^{*}$-algebra with zero center is an example of such an algebra.

The utility of the study of Jordan isomorphisms in Banach algebra was noted by Kadison [8] in the study of isometrics of $B^{*}$-algebras. The Jordan and Lie structures of simple associative rings has been investigated by Herstein in a series of paper (see [3], [4], [5]). Essential use is made of these results in the present work.

2. Pure algebra. Let $R$ be an associative ring. As is well-known [3] we can make $R$ into a Jordan (Lie) ring by introducing the Jordan (Lie) multiplication $x \cdot y=x y+y x([x, y]=x y-y x)$. For a subset $S$ of $R$ we consider the sets

$$
\begin{aligned}
S^{J} & =\{x \in R \mid x \cdot u=0 \text { for all } u \in S\}, \\
S^{L} & =\{x \in R \mid[x, u]=0 \text { for all } u \in S\}, \\
\mathfrak{R}(S) & =\{x \in R \mid u x=0 \text { for all } u \in S\} \text { and } \\
\mathfrak{L}(S) & =\{x \in R \mid x u=0 \text { for all } u \in S\} .
\end{aligned}
$$

By an ideal in $R$ we mean, unless otherwise specified, a two-sided ideal.

2.1. Lemma. Let $U$ be a Lie ideal in $R$. Then $U^{J}$ and $U^{L}$ are Lie ideals.

Let $x \in U^{J}, u \in U$ and $b \in R$. Since $x u=-u x$, an easy computation shows that $[x, b] \cdot u=[b, u] \cdot x=0$. Let $y \in U^{L}$. Since $y u=u y$, we obtain by straightforward calculation that $[[y, b], u]=[y,[b, u]]=0$.

Received June, 23, 1964. This research was supported by the National Science Foundation grant NSF-G-25219. 
2.2. Lemma. Let $I$ be an ideal in $R$. Then

$$
\left[I^{L}, R\right] \subset \mathbb{Q}(I) \cap \mathfrak{R}(I) \subset I^{L} .
$$

Let $y \in I, x \in I^{L}$ and $z \in R$. We get successively the relations $(x z) y=x(z y)=(z y) x=z(y x)=z(x y)=(z x) y$ or $\left[I^{L}, R\right] \subset \mathbb{L}(I)$. Likewise $\left[I^{L}, R\right] \subset \Re(I)$.

For the remainder of this section, $A$ shall denote a semi-prime alegbra over a field of characteristic $\neq 2$. (A semi-prime algebra is one without nilpotent right or left ideals $\neq(0)$ ). It follows from the hypothesis that $A$ is semi-prime, that $\mathscr{L}(I)=\mathfrak{R}(I)$ for any ideal $I$ in $A$ [10] p. 99].

2.3. Lemma. Let $U$ be a Lie ideal in $A$. Then $U^{s}=\mathfrak{Q}(U)=$ $\Re(U)$ is an ideal in $A$ and $U \cap U^{J}=(0)$.

Take $x \in U \cap U^{J}$. Clearly $x^{2}=0$. Also, by Lemma 2.1, $[x, b] \in U \cap U^{J}$ for each $b \in A$. Then

$$
0=[x, b]^{2}=x b x b-x b^{2} x+b x b x .
$$

Multiplying on the left by $x$ and on the right by $b$, we obtain $(x b)^{3}=$ 0 . Thus $x A$ is a nilpotent right ideal so that $x A=(0)$ and hence $x=$ 0 . Therefore $U \cap U^{J}=0$.

Next let $u \in U$ and $x \in U^{J}$. The above shows that $[u, x]=0$ and $u \cdot x=0$. Thus $u x=x u=0$. Hence

$$
U^{J} \subset \mathfrak{Q}(U) \cap \Re(U) .
$$

Next we show that

$$
\mathfrak{L}(U)=\Re(U) \text {. }
$$

For take $x \in \mathcal{L}(U), u \in U$ and $b \in A$. We have $0=x[b, u]=x b u$. Hence $(u x b)^{2}=0$. It follows that $u x=0$ or $\mathfrak{L}(U) \subset \mathfrak{R}(U)$. Likewise $\Re(U) \subset \mathfrak{Q}(U)$. A combination of (1) and (2) gives the desired result. The corresponding proposition for Jordan ideals in harder to prove (see Theorem 2.5).

Let $U$ be a Jordan ideal in $A$. Let $K$ denote the algebraic sum of the ideals in $A$ contained in $U$. Clearly $K$ is maximal in the set of ideals in $A$ contained in $U$.

2.4. Lemma. The ideal $K$ contains $a \cdot b$ and $a A a$ for each $a, b$ of the Jordan ideal $U$ in $A$. If $U \neq(0)$ then $K \neq(0)$.

By a lemma of Herstein [3, Lemma 1], $(a \cdot b) x-x(a \cdot b) \in U$ for each $x \in A$. Since $(a \cdot b) \cdot x \in U$ we see that $(a \cdot b) x$ and $x(a \cdot b)$ lie in $U$. 
If also $y \in A$ then $(a \cdot b) x y+y(a \cdot b) x \in U$. From this we see that the ideal generated by $a \cdot b$ is contained in $U$. Therefore $a \cdot b \in K$. Also $(a \cdot x) \cdot a \in K$. Using $a^{2} \in K$ we obtain $a x a \in K$. If $K=(0)$, then $(a A)^{2}=(0)$ and $a=0$ for all $a \in U$.

2.5. Theorem. Let $U$ be a Jordan ideal in $A$. Then $U^{s}=$ $\mathfrak{L}(U)=\mathfrak{R}(U)$ is an ideal in $A$ and $U \cap U^{J}=(0)$.

Let $u \in U, x \in A$ and $z \in \mathfrak{L}(U)$. We have $0=z(u \cdot x)=(z x) u$. Therefore $\mathfrak{L}(U)$ is an ideal in $A$. Since $[u \mathscr{R}(U)]^{2}=(0)$ we can conclude that $u \mathfrak{R}(U)=(0) \quad$ or $\quad \mathfrak{L}(U) \subset \mathfrak{R}(U)$. Likewise $\mathfrak{R}(U) \subset \mathfrak{L}(U)$. Thus $\mathscr{L}(U) \subset U^{J}$.

Suppose that either $a$ or $b$ lies in $U$. Then

$$
(a \cdot b) \cdot b=a \cdot\left(b^{2}\right)+2 b a b \in U .
$$

In either case it follows that $b a b \in U$. Next let $u \in U, x \in A$. Then $(u \cdot x) \cdot(x u)=u x^{2} u+x u^{2} x+2(x u)^{2} \in U$. By the preceeding remark we see that $(x u)^{2} \in U$. Likewise $(u x)^{2} \in U$. Consider now an ideal $I$ in $A$ such that $I \cap U=(0)$. If $z \in I$ then $(u z)^{2} \in I \cap U=(0)$. Therefore $u I=(0)$. We then have $(0)=U I=I U$.

Let $K$ be the ideal in $A$ maximal in the set of ideals in $A$ contained in $U$ (see Lemma 2.4). In particular $a^{2} \in K$ for $a \in U$. Now $\mathfrak{L}(K)$ is an ideal in $A$. We show that $\mathscr{\&}(K) \cap U=(0)$. For let $b \in \mathbb{L}(K) \cap U, x \in U$. Then $(b x)^{2} \in U \cap \mathbb{R}(K)$. This makes $(b x)^{4}$ an element of $K \cap \mathbb{R}(K)=(0)$. Therefore $(b A)^{4}=(0)$ and $b=0$.

It follows from the above that $U \mathscr{R}(K)=\mathfrak{Q}(K) U=(0)$. Consequently $\mathfrak{L}(K) \subset \mathfrak{L}(U)$. But, inasmuch as $K \subset U$, we have also that $\mathfrak{L}(K)=$ $\mathfrak{L}(U)$. From Lemma 2.3 we get $K^{J}=\mathfrak{L}(K)=\mathfrak{L}(U) \subset U^{J}$. But as $K \subset U$ we see that $K^{J}=U^{J}$. Whereas $U \cap \&(K)=(0)$, we obtain $U \cap U^{J}=(0)$.

2.6. Lemma. If A satisfies the descending chain condition on right ideals, every Jordan ideal in $A$ is an ideal.

By the proof of Theorem 2.5, bab lies in the Jordan ideal $U$ if $a$ or $b$ lies in $U$. It follows from [9, Lemma 5] that $U$ is an ideal.

As we shall see below, Jordan ideals are automatically ideals under favorable conditions. If an algebra $B$ (even a Banach algebra) is not semi-simple, this situation does not prevail and even Lemma 2.6 can fail there, as easy examples show.

For $S, T$ subsets of $A$, by $S \cdot T$ we mean the collection of all finite sums of elements $x \cdot y, x \in S, y \in T$.

2.7. Lemma. Let $I$ be an ideal in $A$. Then $I \cdot A$ is an ideal. 
Also $A \cdot A$ contains $A^{3}$.

The relation $a \cdot(b \cdot c)-(a \cdot b) \cdot c=[[a \cdot c], b]$ is readily verified. Suppose at least one of $a, b$ and $c$ is in $I$. Then $[[a, c], b] \in I \cdot A$. Since $I$ is an ideal, $[a, c] \cdot b \in I \cdot A$. Hence $[a, c] b \in I \cdot A$ and $b[a, c] \in I \cdot A$.

Suppose that $b \in I$. Then $(a c) \cdot b-a[c, b]=(a \cdot b) c \in I \cdot A$. Thus $(a \cdot b) c \in I \cdot A$ and $[b, a] c \in I \cdot A$. Taking the sum and difference of these items, we see that $a b c$ and $b a c \in I \cdot A$ for all $a, c \in A$. Therefore $(a \cdot b) c$ and likewise $c(a \cdot b) \in I \cdot A$ and $I \cdot A$ is an ideal. The proof shows that $A \cdot A \supset A^{3}$.

2.8. Lemma. A maximal ideal $M$ in $A$ is a maximal Jordan ideal.

Let $\pi$ be the natural homomorphism of $A$ onto $A / M$. If $A / M$ is not a zero algebra, then, by [3, Theorem 1], $A / M$ has only itself and (0) as Jordan ideals. Therefore $M$ is a maximal Jordan ideal. Suppose that $A / M$ is a zero algebra. Since its only ideals are trivial, $A / M$ is one-dimensional. This makes $M$ a maximal linear subspace in $A$ and hence a maximal Jordan ideal.

2.9. Theorem. If $A \cdot A=A$, then every maximal Jordan ideal $M$ in $A$ is an ideal.

Let $K$ be the largest ideal in $A$ contained in $M$. If $K$ is a maximal ideal in $A$, then $K=M$ by Lemma 2.8. We show that $K$ is always a maximal ideal. For suppose otherswise. There exists an ideal $I$ in $A, A \neq I, K \subset I, K \neq I$. If $I \supset M$ we are through and $I \subset M$ is impossible. Therefore $A=I+M$. Then $A \cdot M=I \cdot M+M \cdot M$. Lemma 2.4 gives $M \cdot M \subset K \subset I$ so that $A \cdot M \subset I$. Also $A \cdot A=A \cdot I+A \cdot M \subset I$. By hypothesis, this is impossible. In particular, by Lemma 2.7, the conclusion holds if $A^{3}=A$.

2.10. Theorem. Suppose that $A \cdot A=A$ and each ideal in $A$ is the intersection of the maximal ideals containing it. Then every Jordan ideal in $A$ is an ideal.

These conditions are satisfied, for example, if $A$ is biregular in the sense of Arens and Kaplansky [1]. Let $U$ be a Jordan ideal and let $K$ be the largest ideal in $A$ contained in $U$. If $K$ is a maximal ideal, $K=U$ by Lemma 2.8. Suppose that $K$ is not a maximal ideal and let $M$ be any maximal ideal containing $K$. We show that $U \subset M$. For suppose otherwise. Clearly $M \not \subset U$ and $A=M+U$. Reasoning as in the proof of Theorem 2.9, we get $A \cdot A \subset M$, which is impossible. Hence 
$U$ is contained in every maximal ideal $M, M \supset K$. Therefore $U=K$.

3. On topological rings. Now let $A$ be a semi-prime topological algebra over a field of characteristic $\neq 2$.

3.1. THeorem. Suppose that $\mathfrak{Q}(I) \neq(0)$ for every closed ideal $I \neq A$ in $A$. Then any closed Jordan ideal $U$ in $A$ is an ideal in $A$.

As in the preceding section we consider the ideal $K$ in $A$ maximal in the set of ideals contained in $U$. Clearly $K$ is closed. Let $W=$ $K \oplus \mathfrak{L}(K)$. Clearly $x^{2}=0$ for each $x \in \mathfrak{L}(W)=\mathfrak{R}(W)$. Therefore $\mathfrak{L}(W)=(0)$ and $W$ is dense in $A$. Let $a \in U, u \in U$. There exists a net $a_{\alpha}$ in W such that $a_{\alpha} \rightarrow a$. For each $a_{\alpha}, a_{\alpha}=b_{\alpha}+c_{\alpha}$, where $b_{\alpha} \in K$ and $c_{a} \in \mathfrak{L}(K)$. From the proof of Theorem 2.5, $\mathfrak{L}(K)=\mathfrak{L}(U)$. Then we see that $a_{\alpha} u=b_{\alpha} u \in K$ for each $\alpha$. Consequently $a u \in K$. Likewise $u \alpha \in K$, and $U$ is an ideal in $A$.

The condition $\mathfrak{L}(I) \neq(0)$ for proper closed ideals in $A$ gives the natural two-sided analogue of the annihilator algebras of Bonsall and Goldie [2].

3.2. THeOREM. Let $B$ be a semi-simple topological ring. Suppose that

(a) the primitive ideals of $B$ are closed;

(b) $\mathfrak{L}(I) \neq(0)$ for each closed ideal $I \neq A$. Then $B$ is the direct topological sum of its minimal closed ideals.

Note that (a) is automatically satisfied if $B$ is a Banach algebra. Suppose first that $(0)$ is a primitive ideal, i.e., $B$ is primitive. Then (b) and $\mathfrak{L}(I) I=(0)$ for a closed ideal $I$ show that all ideals in $B$ other than (0) must be dense. The desired conclusion is then readily seen. We assume then that $(0)$ is not a primitive ideal.

We call an ideal $K$ in $B$ dual if $\mathfrak{R}(K)=K$. Our first step is to show that each primitive ideal $P$ is dual. There exists a modular maximal right ideal $M$ in $B$ such that $P=\{a \in B \mid x a \in M$ for all $x \in B\}$ and $P$ is the largest ideal in $B$ contained in $M$. Let $j$ be a left identity for $B$ modulo $M$, let $b \in \mathfrak{R} \Re(P)$ and suppose that $b \notin P$. Then there exists an element $z \in B$ such that $z b \notin M$. We can write $j=$ $u+k z b+z b x$ where $u \in M, x \in B$ and $k$ is an integer. Multiplying on the right by an element $w \in \mathfrak{R}(P)=\mathfrak{L}(P)$, we see that $j w=u w \in M$. Since $j w-w \in M$, we also get $w \in M$. Therefore $P \oplus \Re(P) \subset M$. This is impossible since $\mathfrak{R}(P) \neq(0)$ by hypothesis and $P \oplus \mathfrak{R}(P)$ is a larger ideal than $P$ contained in $M$. Hence $P$ is dual.

Next take any ideal $I$ in $B$, where $I \supset P, I \neq P$, for a primitive 
ideal $P$. We show that $\mathscr{L}(I)=(0)$ so that $I$ is dense. Since $I \supset P$, we have $\mathfrak{L}(I) \subset \mathfrak{L}(P)$. Also $(0)=\mathfrak{L}(I) I \subset P$ and $I \not \subset P$. The theory of primitive ideals shows that $\mathfrak{R}(I) \subset P$. Therefore $\mathfrak{L}(I) \subset P \cap \mathbb{L}(P)=(0)$.

Now let $I$ be any dual ideal in $B$. We show that $I$ is the intersection of the primitive ideals in $B$ which contain $I$. Let $\pi$ be the natural homomorphism of $B$ onto $B / I$. Since $I \cap \Re(I)=(0)$, $\pi$ is oneto-one on $\Re(I)$ and $\pi(\Re(I))$ is semi-simple. Let $W$ denote the radical of $B / I$. Then $W \cap \pi(\Re(I))=(0)=W \pi(\Re(I))$ by [6, p. 10]. Therefore $\pi^{-1}(W) \Re(I) \subset I$ from which we see that $\pi^{-1}(W) \Re(I)=(0)$. Thus

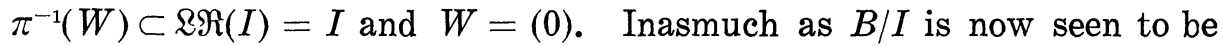
semi-simple, $I$ is the intersection of the primitive ideals which contain it.

Next consider a primitive ideals $P_{0}$ and let $P$ be any primitive ideal, $P \neq P_{0}$. By the above and our hypothesis, $P \not \subset P_{0}$ and $P_{0} \not \subset P$ and $\mathfrak{L}\left(P+P_{0}\right)=(0)$. Therefore $\mathfrak{L}(P) \mathfrak{L}\left(P_{0}\right)=(0)=\mathfrak{L}\left(P_{0}\right) \mathfrak{L}(P)$ so that $\mathfrak{L}\left(P_{0}\right) \subset$ $\mathfrak{R}(P)=P$. Also $\mathfrak{R} \mathfrak{R}\left(P_{0}\right)=\mathfrak{R}\left(P_{0}\right)=\mathfrak{L}\left(P_{0}\right)$ so that $\mathscr{L}\left(P_{0}\right)$ is dual and $\mathcal{L}\left(P_{0}\right) \not \subset P_{0}$. Therefore, as $\mathcal{L}\left(P_{0}\right)$ is the intersection of the primitive ideals containing it, $\mathbb{Q}\left(P_{0}\right)=\cap P$, for $P \neq P_{0}, P$ primitive. It is now clear that the ideals $\mathbb{L}(P), P$ primitive, are the minimal dual ideals of $B$.

View $\mathfrak{L}\left(P_{0}\right)$ as a ring. By [6, p. 206], every primitive ideal of $\mathcal{L}\left(P_{0}\right)$ is the intersection of $\mathscr{L}\left(P_{0}\right)$ with a primitive ideal of $B$. Thus $\mathcal{L}\left(P_{0}\right)$ is a primitive ring. Let $I \neq(0)$ be any closed ideal in $B, I \subset$ $\mathfrak{L}\left(P_{0}\right)$ and let $J$ be the algebraic sum of the ideals $\mathscr{L}(P), P \neq P_{0}, P$ primitive. We claim that $I+J$ is dense in $B$. For let $x \in \Re(I+J)$. Then $x \in \mathfrak{R}(P)=P$ for all $P \neq P_{0}$. Thus $x \in \mathfrak{L}\left(P_{0}\right) \cap \mathbb{R}(I)=K$, say. Since $I K=(0)$ and $\mathbb{E}\left(P_{0}\right)$ is a primitive ring, we get $K=(0)$. By $(b)$, $I+J$ is dense in $B$.

We continue with this notation and show next that $\mathfrak{L}\left(P_{0}\right) \mathfrak{R}\left(P_{0}\right) \subset I$. Let $u \in \mathfrak{L}\left(P_{0}\right), u=\lim \left(v_{\alpha}+q_{\alpha}\right), v_{\alpha} \in I, q_{\alpha} \in J$ and let $x \in \mathfrak{L}\left(P_{0}\right)$. Now each $q_{\alpha} \in P_{0}$ so that $q_{\alpha} x=0$. Therefore $u x=\lim v_{\alpha} x \in I$. This enables us to see that $\mathfrak{L}\left(P_{0}\right)\left(P_{0}+\mathfrak{L}\left(P_{0}\right)\right) \subset I$. Inasmuch as $P_{0}+\mathfrak{L}\left(P_{0}\right)$ is dense in $B$, we get $\mathfrak{L}\left(P_{0}\right) B \subset I$. Likewise $B \mathfrak{L}\left(P_{0}\right) \subset I$. From semi-simplicity we see that $B \mathfrak{Q}\left(P_{0}\right) \neq(0), \mathfrak{R}\left(P_{0}\right) B \neq(0)$. It follows that $\overline{B \mathfrak{Q}\left(P_{0}\right)}=\overline{\mathbb{Q}\left(P_{0}\right) B}$ is a minimal closed ideal in $B$.

For each primitive ideal $P_{\alpha}$, let $Z_{\alpha}=\overline{B_{\mathcal{Q}}\left(\bar{P}_{\alpha}\right)}$. It is clear that if $P_{\alpha} \neq P_{\beta}$, then $Z_{\alpha} \cap Z_{\beta}=(0)=Z_{\alpha} Z_{\beta}$. Then the algebraic sum of the $Z_{\alpha}$ is a direct sum. Let $Q$ be its closure, the direct topological sum of the $Z_{\alpha}$. Note that $\mathfrak{R}\left(Z_{\alpha}\right)=\mathfrak{R}\left(B \mathfrak{L}\left(P_{\alpha}\right)\right)=\mathfrak{R} \mathbb{L}\left(P_{\alpha}\right)=P_{\alpha}$. Therefore $Q=B$. If $I$ is any minimal closed ideal, $I$ must be $Z_{\alpha}$, for some $\alpha$, for otherwise $I$ would annihilate every $Z_{\alpha}$.

3.3. CoRollary. Under the conditions of Theorem 3.2. the primitive ideals of $B$ are the maximal closed ideals of $B$ and every 
ideal in $B$ which is not dense is contained in a primitive ideal. Also $B$ is the direct topological sum of its minimal dual ideals.

Let $I$ be closed ideal in $B, I \neq B$. Then $\mathscr{L}(I) \neq(0)$ and $\mathscr{L}(I) I=$ (0). If $I$ were contained in no primitive ideal we would have $\mathfrak{L}(I)$ contained in every primitive ideal, which is imposible.

The proof of Theorem 3.2 shows that a primitive ideal is a maximal closed ideal. Let $M$ be a maximal closed ideal. As just seen, $M \subset P$ for some primitive ideal $P$, so $M=P$. The proof of Theorem 3.2 also demonstrates that $\mathfrak{L}\left(P_{1}\right) \mathfrak{R}\left(P_{2}\right)=(0)$ if $P_{1}, P_{2}$ are two distinct primitive ideals and that $B$ is the direct topological sum of the $\mathscr{R}(P)$ which are the minimal dual ideals.

4. Continuity of Jordan homomorphisms. We consider a Jordan homomorphism $T$ defined on a Banach algebra $A$ with range a dense subset of a semi-simple Banach algebra $B$. We seek to show that, under reasonable conditions, such a mapping $T$ is automatically continuous. For an element $x$ in $A$ or $B$ we let $\rho(x)$ denote its spectral radius [10, p. 30].

Two useful identities are noted by Kadison [8, p. 330] for the behavior of Jordan homomorphisms relative to Lie products. These are

$$
T([[a, b], c])=[[T a, T b], T c]
$$

and

$$
T\left([a, b]^{2}\right)=[T a, T b]^{2}
$$

for all $a, b, c \in A$. Hence, as noted in [8], $a b=b a$ makes $[T a, T b]$ a central nilpotent element of $B$. Thus, as $B$ is semi-simple, [Ta,Tb] $=0$. Then $T(a b)=T(a b+b a) / 2=T(a) T(b)=T(b) T(a)$.

\subsection{Lemma. For each $x \in A, \rho(T(x)) \leqq \rho(x)$.}

From the above discussion we see that if $a \circ b=b \circ a=0$ that $T(a) \circ T(b)=T(b) \circ T(a)=0$. From this the result is evident.

By the separating set for $T$ we mean the set of $s \in B$ for which there exists a sequence $\left\{x_{n}\right\}$ in $A$ with $\left\|x_{n}\right\| \rightarrow 0$ and $\left\|s-T\left(x_{n}\right)\right\| \rightarrow 0$. By the closed graph theorem, $T$ is continuous if only if $S=(0)$.

Sraightforward arguments show that $S$ is a closed Jordan ideal in $B$.

4.2. Lemma. If $B$ has a left or right identity $j$, then $j \notin S$.

Note that $j$ need not lie in $T(A)$. Suppose that $j \in S$ where $j$ is a left identity for $B$. Then there exists a sequence $\left\{x_{n}\right\}$ in $A$ with 
$\left\|x_{n}\right\| \rightarrow 0$ and $\left\|j-T\left(x_{n}\right)\right\| \rightarrow 0$. For each $z \in B,(z j)^{n}=z^{n} j$. Thus

$$
\left\|(z j)^{n}\right\|^{1 / n} \leqq\left\|z^{n}\right\|^{1 / n}\|j\|^{1 / n} .
$$

From this we see that $\rho(z j) \leqq \rho(z)$. Clearly $\rho(j)=1$. Now $j=$ $(j-z j)+z j$ where the summands permute. Therefore

$$
1=\rho(j) \leqq \rho(j-z j)+\rho(z j) \leqq \rho(j-z j)+\rho(z) .
$$

Now replace $z$ by $T\left(x_{n}\right)$. We see that

$$
\rho\left(j-T\left(x_{n}\right) j\right) \leqq\left\|j-T\left(x_{n}\right)\right\|\|j\| \rightarrow 0
$$

and that, by Lemma $4.1, \rho\left(T x_{n}\right) \leqq\left\|x_{n}\right\| \rightarrow 0$. This yields a contradiction.

4.3. Lemma. Each element of $S$ is a two-sided topological divisor of zero in $B$.

This is a variation on a result of Rickart [10, p. 72]. Let $s \in S$. Arguments used there show that, if $1-\lambda s$ is a two-sided topological divisor of zero for arbitrarily large $\lambda$, then $s$ is a two-sided topological divisor of zero. We assume, then, that $1-\lambda s$ is not a two-sided topological divisor of zero for $|\lambda| \geqq k$. Then $\lambda s$ is quasi-regular, $|\lambda| \geqq k$. Suppose that $s$ is not a left topological divisor of zero in $B$. Then $[10$, p. 24], $B$ has a left identity $j$ and $s$ is a regular elemement of the algebra $j B j$; there exists $u \in j B j$ such that $u s=s u=j$. Then $(u \cdot s) / 2=j \in S$ which is contrary to Lemma 4.2 . Consequently $s$ is a left (and similarly right) topological divisor of zero.

4.4. Lemma. Let $e \neq 0$ be any idempotent in $T(A)$. Then $e \notin S$.

Suppose that $e=T(x), e \in S$. There exists a sequence $\left\{x_{n}\right\}$ in $A$ with $\left\|x_{n}\right\| \rightarrow 0$ and $\left\|e-T\left(x_{n}\right)\right\| \rightarrow 0$. Then also $\left\|e-e T\left(x_{n}\right) e\right\| \rightarrow 0$. From the theory of Jordan homomorphisms (see, for examples [8, p. 329]), $T\left(x x_{n} x\right)=e T\left(x_{n}\right) e$. Now $e-e T\left(x_{n}\right) e$ and $e T\left(x_{n}\right) e$ permute so that, by Lemma 4.1,

$$
\begin{aligned}
1=\rho(e) & \leqq \rho\left(e-e T\left(x_{n}\right) e\right)+\rho\left(e T\left(x_{n}\right) e\right) \\
& \leqq\left\|e-e T\left(x_{n}\right) e\right\|+\rho\left(T\left(x x_{n} x\right)\right) \\
& \leqq\left\|e-e T\left(x_{n}\right) e\right\|+\left\|x x_{n} x\right\| \rightarrow 0 .
\end{aligned}
$$

Therefore $e \notin S$.

If it is not required that the idempotent $e$ be in $T(A)$ the following weaker conclusion holds.

4.5. Lemma. No central idempotent $e \neq 0$ of $B$ lies in $S$. 
We use the notation of the proof of Lemma 4.4. If $e \in S$, then $1=\rho(e) \leqq \rho\left(e-T\left(x_{n}\right)\right)+\rho\left(T\left(x_{n}\right)\right) \leqq\left\|e-T\left(x_{n}\right)\right\|+\left\|x_{n}\right\| \rightarrow 0$.

As an application we take $B=L_{2}(G)$ where $G$ is a compact group and the multiplication in $L_{2}(G)$ is convolution (see [10, p. 330]). Suppose $T(A)$ is dense in $L_{2}(G)$. Lemma 4.5 shows that $S$ cannot contain a central idempotent $\neq 0$. But in $L_{2}(G)$, every nonzero ideal contains a central idempotent. Therefore $S=(0)$ and $T$ is continuous.

4.6. THeOREM. A Jordan homomorphism T of a Banach algebra $A$ onto a dense subset of a strongly semi-simple Banach algebra $B$ is continuous.

Let $M$ be a modular maximal ideal of $B$, and let $\pi$ denote the natural homomorphism of $A$ onto $B / M$. The mapping $\pi T$ is a Jordan homomorphism of $A$ onto a dense subset of the simple algebra $B / M$. The separating set $S_{1}$ for the mapping $\pi T$ is a Jordan ideal of $B / M$ which cannot contain the identity of $B / M$ by Lemma 4.2. But by Herstein's result [3, Theorem 1], $S_{1}$ is an ideal of $B / M$. Therefore $S_{1}=(0)$ and $\pi T$ is continuous. Simple arguments now show that $S \subset M$. Hence $S=(0)$.

4.7. THEOREM. Let $T$ be a Jordan homomorphism of a Banach algebra $A$ onto a semi-simple Banach algebra $B$ where every nonzero right (left) ideal in $B$ contains a minimal right (left) ideal in $B$. Then $T$ is con-tinuous.

Examples of such $B$ are the semi-simple annihilator Banach algebras of Bonsall and Goldie ([10, p. 96] and [2]).

Fix $a, b \in S$. By Lemma 2.4, the ideal generated by $a \cdot b$ lies in $S$. If the right ideal $\{(a \cdot b) x \mid x \in A\} \neq(0)$, it contains an idempotent $e \neq 0$ contrary to Lemma 4.4. Consequently $(a \cdot b) B=(0)$ and $a \cdot b=0$ for all $a, b \in S$. For $a \in S, x \in B$ we have $(a \cdot x) \cdot a=0$. Using $a^{2}=0$ we see that $(a B)^{2}=(0)$. Hence a lies in the radical of $B$ so that $S=(0)$.

4.8. THEOREM. Let $T$ be a Jordan homomorphism of a Banach algebra $A$ onto a dense subset of a primitive Banach algebra $B$ with minimal one-sided ideals. Suppose that $T(A) \cap I$ is dense I for some minimal right (left) ideal $I$. Then $T$ is continuous.

For the case of an algebra homomorphism see [12, p. 378]. Suppose that $I$ is a right ideal. Let $I_{1}=T^{-1}(I)$. If $T\left(x^{2}\right)=0$ for all $x \in I_{1}$, then $u^{2}=0$ for each $u$ in a dense subset of $I$. This would 
make $I^{2}=(0)$ which is impossible. We can express $I=j B$ where $j^{2}=j$. Set $R=\{y \in B \mid j y \in I \cap T(A)\}$. Clearly $R$ is a linear manifold where $j R$ is dense in $j B$. Thus $j R j$ is dense in $j B j$. By the Gelfand-Mazur theorem, $j R j=j B j$. Select $x \in I_{1}$ where $T(x)=j w \neq 0$ and $T\left(x^{2}\right)=$ $j w j w \neq 0$. We have $j w j \neq 0$. Since $j B j$ is a division ring there exists $z \in R$ where $j w j z j=j z j w j=j$. Consequently $(j w) \cdot(j z) \neq 0$. There exists $x_{1} \in I_{1}$ such that $T\left(x_{1}\right)=j z$. Simple computations give $T\left(\left(x \cdot x_{1}\right) / 2\right)=(j w) \cdot(j z) / 2=h$, say, and $T\left[\left(x \cdot x_{1}\right)^{2} / 4\right]=h$. Hence $h$ is an idempotent, $\neq 0$, in $T(A) \cap I$. By Lemma 4.4, $h \notin S$ where $S$ is the separating set for $T$. Consequently $I \not \subset S$.

Denote the socle of $B$ by $\Sigma$ and let $a, b \in S$. By the arguments of Theorem 4.7, the two-sided ideal $Q$ generated by $a \cdot b$ lies in $S$. As $B$ is primitive, $\Sigma \subset Q \subset S$ unless $a \cdot b=0$. Thus, if $a \cdot b \neq(0), h \in S$ which is impossible. Consequently we can reason as in Theorem 4.7 to see that $S=(0)$.

5. Jordan ideals and homomorphisms in $\mathbf{B}^{*}$-algebras. Throughout this section $U$ will denote a closed Jordan ideal in a $B^{*}$-algebra $A$. For an element $x \in A$ which is quasi-regular [10, p. 16] we denote its quasi-inverse by $x^{\prime}$. Since $x \cdot x^{\prime}=\left(x+x^{\prime}\right) / 2$, we see that $x^{\prime} \in U$ if $x \in U$.

\subsection{Lemma. If $h$ is self-adjoint and $h^{2} \in U$, then $h \in U$.}

For all $\alpha>0,\left(-\alpha h^{2}\right)^{\prime}$ exists and lies in $U$. Thus $h \cdot\left(-\alpha h^{2}\right)^{\prime} \in U$. An argument of Rickart [10, Theorem 4.9.2] shows that for any $x \in A$,

$$
x=\lim _{\alpha=\infty} x\left(-\alpha x^{*} x\right)^{\prime}=\lim _{\alpha=\infty}\left(-\alpha x x^{*}\right)^{\prime} x .
$$

Applying this to our case, we obtain $2 h=\lim h \cdot\left(-\alpha h^{2}\right)^{\prime} \in U$.

\subsection{Lemma. $U^{*}=U$.}

Let $a \in U$. By Lemma 2.4, the ideal generated by $a^{2}$ lies in $U$. In particular, $\left[-\alpha\left(\alpha^{2}\right)\left(\alpha^{2}\right)^{*}\right]^{\prime} \in U$ for all $\alpha>0$. By the proof of Theorem 2.5, $\left(a^{2}\right)^{*}\left[-\alpha a^{2}\left(a^{2}\right)^{*}\right]^{\prime}\left(a^{2}\right)^{*} \in U$. Formula (5.1) implies that $\left(a^{4}\right)^{*} \in U$. Since $a^{4} \in U$ it follows that $\left[a^{2}+\left(a^{2}\right)^{*}\right]^{2}=a^{4}+a^{2} \cdot\left(a^{2}\right)^{*}+\left(a^{4}\right)^{*} \in U$, and so, by Lemma $5.1, a^{2}+\left(a^{2}\right)^{*} \in U$. Therefore $\left(a^{2}\right)^{*} \in U$, so that

$$
\left(a+a^{*}\right)^{2}=a^{2}+a \cdot a^{*}+\left(a^{2}\right)^{*} \in U .
$$

Another application of Lemma 5.1 shows that $a+a^{*}$, and thus $a^{*}$, lies in $U$.

5.3. Theorem. Any closed Jordan ideal in a $B^{*}$-algebras $A$ is 
an ideal of $A$.

Let $U$ be a closed Jordan ideal of $A$ and let $a \in U$. Suppose $a=$ $h+i k$, where $h$ and $k$ are self-adjoint. Since $a^{*} \in U$, it follows from Lemma 5.2 that $h \in U$ and $k \in U$. It thus suffices to see that if $h$ and $w$ are self-adjoint and $h \in U$, then $h w$ and $w h$ are in $U$. Now $(w h)^{*}(w h)=h w^{2} h \in U$, since $h \in U$. Also $(w h)(w h)^{*}=w h^{2} w \in U$, since $h \in U$. Consequently for $\alpha>0,\left[-\alpha(w h)^{*}(w h)\right]^{\prime} \in U$, and so $\lim _{\alpha \rightarrow \infty}(w h)\left[-\alpha(w h)^{*}(w h)\right]^{\prime}(w h) \in U$. By formula $(5.1),(w h)^{2} \in U$, and so by Lemma $5.2(h w)^{2} \in U$. Since $U$ is a Jordan ideal, it contains $w h+h w$. But also $[i(w h-h w)]^{2}=-\left\{(w h)^{2}-w h^{2} w-h w^{2} h+(h w)^{2}\right\} \in U$, so again by Lemma $5.1 w h-h w \in U$, and thus $w h$ and $h w \in U$, so $U$ is an ideal.

Automatic continuity occurs for Jordan *-homomorphisms.

5.4. THEOREM. Let $T$ be a Jordan *-homomorphism of a $B^{*-}$ algebra $A$ onto a dense subset of a $B^{*}$-algebra $B$. Then

(a) $T$ is continuous and $\|T\|=1$,

(b) the range of $T$ is $B$, and

(c) the adjoint mapping $T^{\prime}$ is an isometry.

Before proceeding with the proof we should remark that these are instances of positive linear maps on operator algebras, which have been extensively studied. See [15].

Let $A_{0}$ be the closed $*$-subalgebra generated by a self-adjoint element $h \in A$. Then $T$ is a $*$-homomorphism of the commutative $B^{*}$ algebra $A_{0}$ into $B$. Therefore $\|T(h)\| \leqq \rho(T(h)) \leqq \rho(h) \leqq\|h\|$. Consequently if $h$ and $k$ are any two self-adjoint elements of $A$,

$$
\begin{aligned}
\|T(h+i k)\| & \leqq\|T(h)\|+\|T(k)\| \leqq\|h\|+\|k\| \\
& \leqq\|h+i k\|+\|h-i k\|=2\|h+i k\| .
\end{aligned}
$$

Thus $T$ is continuous.

Hence the kernel $T^{-1}(0)$ of the mapping $T$ is a closed Jordan ideal in $A$. By Theorem 5.3, $T^{-1}(0)$ is an ideal and [10, p. 249] $A / T^{-1}(0)$

is a $B^{*}$-algebra in the quotient space norm. Let $T_{0}$ be the mapping of $A / T^{-1}(0)$ onto $B$ defined by $T_{0}\left(x+T^{-1}(0)\right)=T(x)$ and let $\pi$ be the natural homomorphism of $A$ onto $A / T^{-1}(0)$. The mapping $T_{0}$ restricted to the closed $*$-subalgebra of $A / T^{-1}(0)$ generated by a normal element is an isometry by [10, p. 241]. That $T_{0}$ is an isometry on normal elements allows us to use the arguments of [8, Theorem 5] to assert that $T_{0}$ is an isometry on $A / T^{-1}(0)$. The range of $T_{0}$ and therefore that of $T$ is then all of $B$, and $\|T(x)\|=\|\pi(x)\|$ for all $x \in A$. Since a natural homomorphism has norm one, $\|T\|=1$. Now $T=T_{0} \pi$, so 
$T^{\prime}=\pi^{\prime} T_{0}^{\prime}$. As each of $T_{0}^{\prime}$ and $\pi^{\prime}$ is an isometry by the theory of normed linear spaces, so is $T^{\prime}$.

5.5. THeOREM. Let $A, B$ be $B^{*}$-algebras with identities $e_{A}$ and $e_{B}$, respectively. The Jordan *-homomorphisms of $A$ onto $B$ are precisely the continuous linear mappings $T$ of $A$ onto $B$ such that (a) the adjoint $T^{\prime}$ is isometric, (b) the kernel of $T$ is an ideal of $A$, and $(c) T\left(e_{A}\right)=c_{B}$.

By Theorem 5.4, any Jordan *-homomorphism of $A$ onto $B$ has properties (a), (b) and (c).

Suppose $T$ has the properties (a), (b), and (c). Let $\pi$ be the natural homomorphism of $A$ onto $A / T^{-1}(0)$. Let $T_{0}$ be as in the prior theorem. Then $T=T_{0} \pi$, and $T^{\prime}=\pi^{\prime} T_{0}^{\prime}$. Since $T^{\prime}$ is an isometry, so is $T_{0}^{\prime}$. As $T_{0}$ is a one-to-one mapping of $A / T^{-1}(0)$ onto $B, T_{0}^{\prime}$ is an isometry of the conjugate space of $B$ onto the conjugate space of $A / T^{-1}(0)$. Thus we conclude that $T_{0}$ is an isometry of $A / T^{-1}(0)$ onto $B$. Since $T_{0}\left(e_{A}+T^{-1}(0)\right)=e_{B}$, a result of Kadison $[8, \mathrm{p} .330]$ shows that $T_{0}$ is a Jordan $*$-isomorphism and thus that $T=T_{0} \pi$ is a Jordan *-homomorphism.

In [8, p. 329] Kadison shows that if $T$ is a Jordan $*$-isomorphism of a $B^{*}$-algebra $A$ onto a $B^{*}$-algebra $B$, then $T$ is an isometry. The conventions of that paper require a unit for the algebra. However, a check of the argument involved in the proposition quoted above shows that the identity plays no role whatsoever. The next proposition is in the nature of a converse statement.

5.6. TheOREM. Let $A$ be a $B^{*}$-algebra, and $B$ a Banach algebra with an involution. Let $T$ be a Jordan *-isomorphism of $A$ onto $B$ which is an isometry. Then $B$ is a $B^{*}$-algebra.

Let $x \in A$. Then $\left\|(T x)^{n}\right\|=\left\|T x^{n}\right\|=\left\|x^{n}\right\|$, so $\rho(T x)=\rho(x)$ for all $x \in A$, where $\rho$ designates the spectral radius in either algebra.

Suppose $T x=y \in \operatorname{Rad} B$, the radical of $B$. Then $y^{*} \in \operatorname{Rad} B$ and consequently $\rho\left(y \pm y^{*}\right)=0=\rho\left(x \pm x^{*}\right)$. But $x+x^{*}$ and $x-x^{*}$ are selfadjoint and skew elements in a $B^{*}$-algebra, so $x \pm x^{*}=0$. Consequently $x=0=y$, and thus $B$ is semi-simple.

It then follows (see $\S 4$ ) that the spectrum of $T(x)$ if the same as that of $x$. Consequently, if $u \in B$ and $u$ is self-adjoint, then its spectrum is real and $\rho(u)=\|u\|$. A result of Yood [13, p. 148] now asserts that there is a bicontinuous $*$-isomorphism $\sigma$ of $B$ onto a $B^{*}$ algebra $B_{1}$. The mapping $\sigma T$ of $A$ onto $B_{1}$ is a Jordan $*$-isomorphism. 
By the quoted result of Kadison, $\sigma T$ is an isometry. Since $T$ is an isometry, so is $\sigma$ and, therefore, $B$ is a $B^{*}$-algebra.

We use the terminology Jordan involution for a conjugate linear mapping $x \rightarrow x^{*}$ of period two on a semi-simple complex Banach algebra $A$ where $(x \cdot y)^{*}=x^{*} \cdot y^{*}, x, y \in A$. We write $H=\left\{x \in A \mid x=x^{*}\right\}$. Clearly $A=H \oplus i H$ and $\rho\left(x^{*}\right)=\rho(x), x \in A$.

5.7. Lemma. Suppose that there is a real normed linear space norm $|x|$ on $H$ such that $|x| \leqq \rho(x), x \in H$. Then the Jordan involution $x \rightarrow x^{*}$ is continuous.

Let $H^{\prime}$ be the derived set of $H$. Note that $y^{2} \in H$ if $y \in H$. The arguments of [11, Lemma 3.3] show $y^{2}=0$ if $y \in(i H) \cap H^{\prime}$. Thus $\rho(y)=\rho(i y)=0$ and $y=0$ for such $y$. It follows [11, p. 157] that $H$ is closed. If $x \in A$ and $x=u+i v, u, v \in H$ the norm $\|x\|_{1}=\|u\|+\|v\|$ is then a complete linear space norm for $A$ topologically equivalent to the given norm so that the Jordan involution is continuous.

We are now able to establish the continuity of a Jordan homomorphism of a complex Banach algebra $A$ onto a $B^{*}$-algebra. There need be no involution on $A$.

5.8. THEOREM. Let $T$ be a Jordan homomorphism of a complex Banach algebra $A$ onto a $B^{*}$-algebra $B$. Then $T$ is continuous.

Let $P_{\alpha}$ be the set of primitive ideals of $B$, and let $\pi_{\alpha}$ be the canonical homomorphism of $B$ onto $B / P_{\alpha}$. Then $\pi_{\alpha} T$ is a Jordan homomorphism of $A$ onto a primitive algebra. By a result of Herstein [4, p. 340], $\pi_{\alpha} T$ is either a homomorphism or an anti-homomorphism. Since $B / P_{\alpha}$ is a $B^{*}$-algebra, in either case we see that $\pi_{\alpha} T$ is continuous [10, Theorem 4.1.20]. This shows that $\left(\pi_{a} T\right)^{-1}(0)$ is a closed ideal in $A$. But

$$
T^{-1}(0)=\bigcap_{\alpha}\left(\pi_{\alpha} T\right)^{-1}(0) \text {. }
$$

Therefore $T^{-1}(0)$ is a closed ideal in $A$. It is not difficult to show that $A / T^{-1}(0)$ is semi-simple.

Let $\pi$ denote the natural homomorphism of $A$ onto $A / T^{-1}(0)$ and let $T_{0}$ be the Jordan isomorphism $T_{0}(\pi(x))=T(x)$ of $A / T^{-1}(0)$ onto $B$. By $\S 4$ we see that $\rho[T(x)]=\rho\left[T_{0}(\pi(x))\right]=\rho[\pi(x)]$ since $B$ is semi-simple.

The natural involution $x \rightarrow x^{*}$ on $B$ induces a Jordan involution on $A / T^{-1}(0)$ by the rule $\left[T_{0}^{-1}(w)\right]^{*}=T_{0}^{-1}\left(w^{*}\right)$. Suppose that $T_{0}^{-1}(h)$ is selfadjoint under this Jordan involution. Then $T_{0}^{-1}(h)=\left[T_{0}^{-1}(h)\right]^{*}=T_{0}^{-1}\left(h^{*}\right)$ so that $h=h^{*}$ and $h$ is self-adjoint in $B$. Thus $\rho\left(T_{0}^{-1}(h)\right)=\rho(h)=\|h\|$ for all $h$ self-adjoint in $B$ and $\left|T_{0}^{-1}(h)\right|=\|h\|$ serves as the auxiliary norm needed for Lemma 5.7. By that result, the Jordan involution 
is continuous on $A / T^{-1}(0)$.

To show that $T$ is continuous it is enough to show that $T_{0}$ is continuous. For the continuity of $T_{0}$ it suffices to show that $\left\|\pi\left(x_{n}\right)\right\| \rightarrow 0$ and $\left\|T_{0}\left(\pi\left(x_{n}\right)\right)-T_{0}\left(\pi\left(x_{0}\right)\right)\right\| \rightarrow 0$ imply that $T_{0}\left(\pi\left(x_{0}\right)\right)=T\left(x_{0}\right)=0$. We can write $\pi\left(x_{n}\right)=\pi\left(u_{n}\right)+i \pi\left(v_{n}\right), n=0,1,2, \cdots$, where each $\pi\left(u_{n}\right)$ and $\pi\left(v_{n}\right)$ is self-adjoint in terms of the Jordan involution of $A / T^{-1}(0)$. Since that involution is continuous, $\left\|\pi\left(u_{n}\right)\right\| \rightarrow 0$ and $\left\|\pi\left(v_{n}\right)\right\| \rightarrow 0$. Each $T_{0}\left(\pi\left(u_{n}\right)\right)=T\left(u_{n}\right)$, and each $T\left(v_{n}\right)$, is self-adjoint in the $B^{*}$-algebra $B$. Thus $\left\|T\left(u_{n}\right)-T\left(u_{0}\right)\right\| \rightarrow 0$ and $\left\|T\left(v_{n}\right)-T\left(v_{0}\right)\right\| \rightarrow 0$. Also

$$
\begin{aligned}
\rho\left(T\left(u_{0}\right)\right) & =\left\|T\left(u_{0}\right)\right\| \leqq\left\|T\left(u_{n}\right)-T\left(u_{0}\right)\right\|+\left\|T\left(u_{n}\right)\right\| \\
& =\left\|T\left(u_{n}\right)-T\left(u_{0}\right)\right\|+\rho\left(T\left(u_{n}\right)\right) \\
& =\left\|T\left(u_{n}\right)-T\left(u_{0}\right)\right\|+\rho\left(\pi\left(u_{n}\right)\right) \rightarrow 0 .
\end{aligned}
$$

Therefore $T\left(u_{0}\right)=0$ so that $\pi\left(u_{0}\right)=0$. Likewise $\pi\left(v_{0}\right)=0$ and thus $\pi\left(x_{0}\right)=0$.

6. Lie ideals in Banach algebras. Throughout let $B$ be a complex Banach algebra with center 3. Suppose that $B$ is semi-simple. If $e^{2}=e$ and $e B(B e)$ is a minimal right (left) ideal of $B$, we call $e$ a minimal idempotent of $B$. By the Gelfand-Mazur Theorem, $e B e=K e$, where $K$ is the set of complex numbers. If $B$ is primitive, it is known $[10$, p. 61$]$ that either $3=(0)$ or $B$ has an identity $u$ and $3=K u$.

Consider the Lie multiplication $[x, y]=x y-y x$ in $B$. In this topological algebraic setting we modify standard notation [7] as follows. Let $[S, T]$ be the closed linear span of the elements $[s, t]$ where $s \in S$, $t \in T$ and $S, T$ are subsets of $B$. If $\&$ is a Lie ideal of $B$ we define $D^{k} \Omega$ inductively by $D^{\circ} \mathbb{Q}=\overline{\mathfrak{Q}}$ and $D^{k+1} \Omega=\left[D^{k} \Omega, D^{k} \Omega\right]$. A Lie ideal $\mathbb{Q}$ is called solvable if for some positive integer $k, D^{k} \Omega=(0)$. The Lie radical of $B$ is defined to be the closed linear span of all the solvable Lie ideals of $B$.

As in [3] we define $S(\mathfrak{Q})=\{x \in B \mid[x, y] \in \mathfrak{Q}$ for all $y \in B\}$.

6.1. THEOREM. Let $B$ be a primitive Banach algebra with socle $S \neq(0)$. Let $e$ be a minimal idempotent and $\mathbb{Q} \neq(0)$ be a Lie ideal of $B$. Then

(a) $e \Omega e=K e$.

(b) If $[\Omega, \Omega]=(0)$, then $\mathbb{Q}=3$.

(c) If $e \in \mathbb{R}$, then $\mathbb{R} \supset S$.

(d) If $\mathbb{Q} \neq 3$, then $\&$ contains $[x, y]$ for all $x \in S, y \in B$.

(a) Suppose that $e \Omega e \neq K e$. Then $e \Omega e \doteq(0)$. Let $a \in \Re$ and $x \in B$. We have $e(a x-x a) e=0$ or eaxe $=e x a e$. Replacing $x$ by $x e$, we see that $e a x e=0$. This gives $(e a x)^{2}=0$ for all $x \in B$, so that $e a=0$. 
Then $e(a x-x a)=0=e x a$. From this we get $(B e B)(B a B)=(0)$. As $B$ is primitive it follows that $a=0$. This makes $\mathbb{L}=(0)$.

(b) For any $x \in \mathbb{R}, y \in B$ we have $\left[x, y^{2}\right]=[x, y] \cdot y \in \mathbb{L}$. Since $[[x, y], y] \in \mathbb{R}$. it follows that $(x y-y x) y$ and $y(x y-y x) \in \mathbb{R}$. As $[\Omega, \Omega]=(0), x$ permutes with these elements and with $x y-y x$. Therefore $(x y-y x) y x=(x y-y x) x y$ or $(x y-y x)^{2}=0$. Since $e(x e-e x)^{2} e=0$, we obtain $e x^{2} e=(e x e)^{2}, x \in \mathfrak{L}$. Replacing $x$ by $x y-y x$ we see that $[e(x y-y x) e]^{2}=0$. Since $e B e=K e$, we have $e[\Omega, B] e=(0)$. But $[\Omega, B]$ is a Lie ideal so that, by (a), $\& \subset 3$. But 3 is one-dimensional or (0). whence $\mathfrak{L}=3$.

(c) Given $e \in \mathfrak{R}$, we have $[e,[e, x]] \in \mathbb{Z}$ for all $x \in B$. Inasmuch as exe is a scalar multiple of $e$, we see that $e \cdot x \in \mathfrak{Z}$. As $[e, x] \in \mathbb{R}$, we get $e x \in \mathfrak{Z}$ and $[y, e x] \in \mathbb{Q}$ for all $x, y \in B$. But $[y, e x]=y e x-e x y$ so that $B e B \subset \Omega$. By [6, p. 75] we see that $\mathscr{S} S$.

(d) By [3, p. 282], $S(\Omega)$ is both a Lie ideal and a subalgebra of

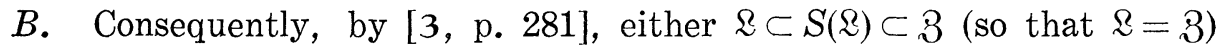
or $S(\mathbb{R})$ contains a nonzero ideal of $B$. In the latter case, $S(\mathbb{R}) \supset S$ since $B$ is primitive. This yields (d).

6.2. THEOREM. Let $B$ be a primitive Banach algebra with minimal one-sided ideals. Then the Lie radical of $B$ coincides with 3.

It is sufficient to show that any solvable Lie ideal $\&$ of $B$ is contained in 3. Suppose $D^{\circ} \mathbb{Q}=\overline{\mathrm{Z}}$ and $D^{p+1} \Omega=\left[D^{p} \Omega, D^{p} \Omega\right], p=0,1, \cdots$, where $k$ is the smallest integer such that $D^{k} \Omega=(0)$. We may suppose $k \geqq 1$ and then $\left[D^{k-1} \Omega, D^{k-1} \Omega\right]=(0)$. By Theorem 6.1 , we see that $D^{k-1} Q \subset 3$. If $k=1$ we are through. Otherwise $\left[D^{k-2} \Omega, D^{k-2} \Omega\right] \subset 3$. If $3 \neq(0)$ then $3=K u$ where $u$ is the identity of $B$. But for $x, y \in D^{k-2} Q, x y-y x=\alpha u, \alpha \neq 0$ is impossible. For if this relation persists then $s p(x y)=\alpha+s p(y x)$ whereas $s p(x y)$ and $s p(y x)$ agree up to the single value $\{0\}$ and are compact sets. Therefore $\left[D^{k-2} \Omega, D^{k-2} \Omega\right]=$ $D^{k-1} \mathfrak{Q}=(0)$ and $D^{k-2} \mathbb{C} \subset 3$. This argument, repeated a finite number of times, leads to $R \subset Z$.

6.3. CoRollary. Let $B$ be a semi-simple Banach algebra with socle $S \neq(0)$ where $S$ is contained in no primitive ideal of $B$. Then the Lie radical of $B$ is contained in 3 .

Let $\&$ be a solvable Lie ideal of $B, P$ be a primitive ideal and $\pi$ be the natural homomorphism of $B$ onto $B / P$. By hypothesis there exists a minimal idempotent $e$ of $B, e \notin P$. Now $e B e=K e$ so that $\pi(e) \pi(B) \pi(e)=K \pi(e)$ from which it follows that $\pi(e)$ lies in the socle 
of $B / P$. Now $\pi(\Omega)$ is a solvable Lie ideal of $B / P$ so that, by Theorem 6.2 , either $\pi(\mathbb{R})=(0)$ or $\pi(\mathbb{Q})=K \pi(u)$ where $\pi(u)$ is an identity for $B / P$. Hence, for $x \in \mathfrak{R}$, either $x \in P$ or we can write $x=\alpha u+y$, $\alpha \in K, y \in P$. In either case we get $[x, w] \in P$ for all $w \in B$. There-

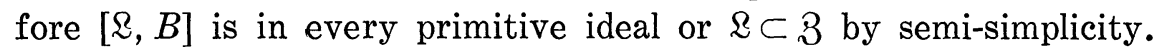

There is an additional fact relating to primitive ideals which will be useful in the sequel.

6.4. Lemma. Let $P$ be a primitive ideal of a Banach algebra $B$. Then either $S(P)=P$ or $S(P)=K u+P$ where $u$ is an identity for $B$ modulo $P$.

Let $\pi$ be the natural homomorphism of $B$ onto $B / P$. Let $x \in S(P)$ and $y \in B$. Then clearly $\pi(x) \pi(y)=\pi(y) \pi(x)$ or $\pi(x)$ lies in the center $B_{1}$ of $B / P$. Now $B / P$ is primitive so either $B_{1}=(0)$ or $B / P$ has an identity $\pi(u)$ and $B_{1}=K \pi(u)$. If $B_{1}=(0)$ clearly $S(P)=P$. If $3_{1}=$ $K \pi(u)$ then $P \subset S(P) \subset K u+P$ so that either $P=S(P)$ or $K u+P=$ $S(P)$.

7. Annihilation and Lie annihilation. In Theorem 3.2 and Corollary 3.3 we obtained structure theorems for a class of algebras where, for each proper closed ideal $I, \mathfrak{L}(I) \neq(0)$. In this section we consider the consequences of a weaker hypothesis on the proper closed ideals for Banach algebras.

Before we investigate the weakened hypothesis, let us note the implication of the statement $\mathfrak{L}(I)=(0)$. In Lemma 2.2, we saw that if $I$ is an ideal in a ring $A$, then $\left[I^{L}, A\right] \subset \mathbb{R}(I) \cap \Re(I) \subset I^{L}$. Thus if $\mathfrak{L}(I)=(0), I^{L}=3$, the center of $A$. Suppose that $A$ is semi-prime (so that $\mathfrak{L}(I)=\mathfrak{R}(I)$ ) and that 3 contains no nonzero ideal of $A$. Then if $I^{L}=3, \mathfrak{L}(I)=(0)$. Hence for many of the algebras under consideration in this paper, $I^{L}=3$ if and only if $\&(I)=(0)$.

Throughout this section, $B$ denotes a semi-simple Banach algebra, with center 3 , satisfying the condition:

(7.1) If $\mathfrak{Q}(I)=(0)$, for an ideal $I$, then $I+3$ is dense in $B$.

Since $\mathfrak{L}(I+\mathfrak{R}(I))=(0)$, an immediate consequence of (7.1) is that for every ideal $I, I+\Re(I)+3$ is dense in $B$. Also, since $I+3 \subset S(I)$, it is immediate that $\Re(I)+S(I)$ is dense in $B$.

7.1. Lemma. Let $I$ be a closed ideal of $B$. Then $\Re \Re(I) \subset S(I)$.

Let $x \in \mathfrak{R}(I), y \in B$. We can write $y=\lim \left(u_{n}+v_{n}+z_{n}\right)$ where $u_{n} \in I, v_{n} \in \mathfrak{R}(I)$ and $z_{n} \in 3$. Then $x y-y x=\lim \left(x u_{n}-u_{n} x\right) \in I$. 
Therefore $x \in S(I)$.

7.2. Lemma. A primitive ideal $P$ for which $\mathfrak{L}(P) \neq(0)$ is a ideal. Also $\mathfrak{L}(P)$ is a minimal dual ideal.

The first statement is shown by the proof of Theorem 3.2. Let $I \neq(0), I \subset \mathbb{2}(P)$, where $I$ is dual. Since $I \not \subset P$ and $P$ is a primitive ideal we see that $\mathfrak{L}(I) \subset P$. Thus $I=\mathfrak{R}(I) \supset \mathfrak{L}(P)$ which forces $I=$ $\mathfrak{L}(P)$.

7.3. Lemma. Let $P$ be a primitive ideal of $B$ for which $\&(P)=$ (0). Then $P$ is a modular maximal ideal of $B$ of deficiency one, and there is an identity for $B$ modulo $P$ which is central.

Proof. By (7.1), $P+3$ is dense. Let $\pi$ be the natural homomorphism of $B$ onto $B / P$. Then $\pi(P+3)=\pi(3)$ is dense in $B / P$. Therefore $B / P$ is a commutative primitive Banach algebra, i.e., a field. Thus there exists $u \in 3$ such that $B=P+K u$. Clearly $u$ can be selected to be an identity for $B$ modulo $P$.

7.4. THEOREM. Let $B$ be a semi-simple Banach algebra satisfying (7.1). Let $I$ be the smallest dual ideal of $B$ which contains all the minimal dual ideals of $B$, then $B / I$ is commutative.

Let $J$ be the algebraic sum of all the ideals $\Re(P) \neq(0)$ for $P$ primitive. By Lemma 7.2, $I \supset J$. Also clearly $\Re(J)=\cap P$, for the primitive ideals with $\Re(P) \neq(0)$.

Let $W=\cap M$, where $M$ is a maximal ideal of deficiency one with a relative identity in 3. For any maximal modular ideal of the stated form $B=M+K u$, with $u \in 3$, so $[B, B] \subset M$. Thus $[B, B] \subset W$. By (7.1), Lemma 7.3, and semi-simplicity, $W \cap \Re(J)=(0)$, so $W \subset \Re \Re(J) \subset I$. Thus $[B, B] \subset I$, and consequently $B / I$ is commutative.

We next consider a hypothesis on the Lie ideals which will enable us to obtain results on the Lie structure of certain Banach algebras.

(7.2) If $\mathfrak{Q}$ is a closed Lie ideal of $B$ and $\mathbb{Q} \supset \mathbb{R}^{L}$, then $\mathfrak{Q}=B$.

7.5. THEOREM. Let $B$ be a semi-simple Banach algebra which satisfies (7.2). Then $B$ satisfies the conclusion of Theorem 7.4.

We show that if (7.2) is satisfied so is (7.1). In view of Lemma 2.2 , if $\mathfrak{L}(I)=0$ for an ideal $I$ then $\left[I^{L}, B\right]=(0)$ or $I^{L} \subset 3$. Thus if $\mathfrak{\Im}=I+3, \mathfrak{\Im}$ is a Lie ideal and $\mathfrak{3} \supset I^{L}=\mathfrak{\Im}^{L}$. Consequently (7.2) shows that (7.1) is satisfied. 
If the semi-simple Banach algebra $B$ satisfies (7.2) and also

\section{(7.3) B has zero center}

then (7.1) is satisfied with $3=(0)$, so we are with in the framework of $\S 3$, and in particular Corollary 3.3 holds. Thus a structure holds in terms of minimal associative objects. We next turn our attention to obtaining a structure theorem under these hypotheses in terms of certain Lie ideals.

Any $H^{*}$-algebra [10, pp. 272-276] $B$ with zero center satisfies (7.2) and (7.3). Let $\&$ be a closed Lie ideal of $B$, and suppose that $\& \supset \mathbb{R}^{L}$. Let $I$ be any minimal closed ideal of $B$. Direct calculation with the " matrix" representation of $I$, shows that the only closed Lie ideals in $I$ are $(0)$ and $I$. Hence $[\Omega, I]=(0)$ or $[2, I]=I$. In the first instance $I \subset \mathbb{Q}^{L} \subset \mathbb{R}$, and in the second $I \subset \mathcal{Q}$ directly. Thus for all minimal closed ideals of $B, \& \supset I$, so $\&=B$ and (7.2) is satisfied.

Until otherwise is stated we assume $B$ is a semi-simple Banach algebra satisfying (7.2) and (7.3).

\subsection{Lemma. $B$ is Lie semi-simple.}

It is sufficient to show that $[\mathbb{R}, \mathbb{R}]=(0)$ for a Lie ideal $\mathbb{R}$ of $B$ implies $\mathbb{L}=(0)$. Now $[\mathbb{Q}, \mathbb{R}]=0$ implies $\mathbb{Q} \subset \mathbb{R}^{L}$, and thus that $\mathbb{2}^{L L} \subset \mathbb{R}^{L}$. Condition (7.2) then shows that $\mathbb{R}^{L}=B$, whence $\mathbb{C} B=(0)$ by (7.3).

The Lie semi-simplicity will frequently be used in the form that $\mathfrak{Q} \cap \mathbb{R}^{L}=(0)$ for any Lie ideal $\mathbb{\&}$ of $B$.

\subsection{CoRollary. $B$ contains no modular two-sided ideals.}

If $M$ were a maximal modular ideal of $B$, then (7.1) implies $B=$ $M \oplus \mathfrak{L}(M)$. Let $e$ be an identity for $B$ modulo $M$, with $e \in \mathfrak{L}(M)$. Clearly $e \in(\mathscr{L}(M))^{L} \cap \mathbb{L}(M)=(0)$ by the prior lemma. Thus we have a contradiction and there can be no modular ideals in $B$.

7.8. Lemma. If $\mathbb{Q}$ is a Lie ideal of $B$, then $S\left(\mathbb{Q}^{L}\right)=\mathfrak{Q}^{L}$ and $S\left(L^{-}\right)=\mathfrak{Q}^{L L}$.

Clearly $L^{L} \subset S\left(\mathbb{Q}^{L}\right)$. Let $x \in S\left(\mathbb{Q}^{L}\right)$ and $y \in \mathbb{R}$. Then $[x, y] \in \mathbb{R}^{L} \cap \mathbb{R}=$ (0), so $S\left(\mathbb{R}^{L}\right) \subset \mathbb{Q}^{L}$.

Since $\mathfrak{Q} \subset \mathbb{Q}^{L L}$ and the latter is closed, $S\left(\mathcal{Q}^{-}\right) \subset S\left(\mathbb{Q}^{L L}\right)=\mathfrak{Q}^{L L}$ by the above. Now (7.2) implies that $\mathbb{Q}+\mathbb{2}^{L}$ is dense in $B$. Let $x \in \mathbb{R}^{L L}$. Then if $y \in B, y=\lim \left(y_{j}+z_{j}\right)$ with $y_{j} \in \mathbb{R}$ and $z_{j} \in \mathbb{R}^{L}$. Consequently, $[x, y]=$ $\lim \left[x, y_{j}+z_{j}\right]=\lim \left[x, y_{j}\right] \in \mathbb{L}^{-}$, and so $\mathfrak{R}^{L L} \subset S\left(\mathbb{R}^{-}\right)$. 
7.9. Corollary. If $P$ is a primitive ideal of $B$, then $p=p^{L L}$.

It follows from Lemma 6.4 that $S(P)=P$ unless $P$ is a modular ideal. However, by Corollary 7.7 there are no modular ideals, so Lemma 7.8 yields the desired result.

7.10. Lemma. If $I$ is a closed ideal in $B$, then $(\Re(I))^{L}=S(I)$.

Since $\Re(I) \subset I^{L}$, it follows from Lemma 7.8 that $S(I)=I^{L L} \subset(\Re(I))^{L}$. Let $x \in(\Re(I))^{L}$ and $y \in B$. Since the ideal $I+\Re(I)$ is dense in $B, y=$ $\lim \left(p_{n}+q_{n}\right)$, with $p_{n} \in I$ and $q_{n} \in \Re(I)$. Then

$$
[x, y]=\lim \left[x, p_{n}+{ }_{q_{n}}\right] \lim \left[x, p_{n}\right] \in I,
$$

and consequently $(\Re(I))^{L} \subset S(I)$.

7.11. Lemma. Let $P$ be a primitive ideal of $B$. Then $[\Re(P)$, $\Re(P)]$ is contained in every nonzero closed Lie ideal in $\Re(P)$.

Let $\Im \neq(0)$ be a closed Lie ideal of $B$ with $\Im \subset \Re(P)$. By the use of two results of Herstein, we see that $S(\mathfrak{\Im})$ is a ring as well as a Lie ideal [3, p. 282] and therefore [3, p. 281] that either $S(\Im)$ is in the center of $B$ or contains a nonzero ideal $I$ of $B$. The first possibility cannot pertain here since (7.3) holds. Thus we have a nonzero ideal $I \subset S(\Im)=\Im^{L L} \subset(\Re(P))^{L L}=P^{L}$ by use of Lemmas 7.8, 7.10 and Corollary 7.9. This implies that $\mathfrak{R}(\Re(I)) \cdot \Re(P) \neq 0$, for otherwise $I \subset \mathfrak{R}(\Re(I)) \subset P=\mathfrak{L}(\Re(P))$, and thus $I \subset P \cap P^{L}=(0)$, which is a contradiction. Consequently $\mathscr{R}(\Re(I)) \cap \Re(P)$ is a dual ideal $\neq(0)$ contained in the minimal dual ideal $\Re(P)$. Therefore $\Re(P) \subset \Re \Re(I)$ and $\Re(I) \subset P$.

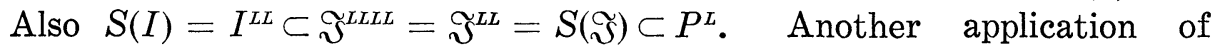
Lemma 7.10 yields $P^{L} \subset(\Re(I))^{L}=S(I)$. However, from $I \supset P^{L}$, we deduce $S(I)=I^{L L} \subset P^{L}$, so $P^{L}=S(I)=S(\Im)$. Let $D$ be the largest ideal contained in $P^{L}$. The Theorem 1 in [14, p. 156] together with its proof shows that $\mathfrak{[}[D, B]$. Since $D \supset \Re(P), \mathfrak{\Im} \supset[\Re(P), \Re(P)]$.

7.12. Lemma. For any primitive ideal $P$ of $B,[\Re(P), \Re(P)]^{L}=P$.

The inclusion $P \subset[\Re(P), \Re(P)]^{L}$ is immediate. Let $x \in P^{L}=(\Re(P))^{L L}$ by Lemmas 7.9 and 7.10. For $y \in B, y=\lim \left(p_{n}+r_{n}\right)$ with $p_{n} \in P$ and $r_{n} \in \mathfrak{R}(P)$, and $x=\lim \left(s_{m}+t_{m}\right)$ with $s_{m} \in P$ and $t_{m} \in \mathfrak{R}(P)$. Thus

$$
\begin{aligned}
{[x, y] } & =\lim _{n}\left[x, p_{n}+r_{n}\right]=\lim _{n}\left[x, r_{n}\right]=\lim _{n} \lim _{m}\left[s_{m}+t_{m}, r_{n}\right] \\
& =\lim _{n} \lim _{m}\left[t_{m}, r_{n}\right] \in[\Re(P), \Re(P)] .
\end{aligned}
$$


Consequently $\left.P^{L} \subset S([P), \Re(P)]\right)=[\Re(P), \Re(P)]^{L L}$, and so $[\Re(P), \Re(P)]^{L} \subset$ $P^{L L}=P$. We thus have the asserted equality.

7.13. Lemma. Each primitive ideal $P$ of $B$ is a maximal proper closed Lie ideal of $B$.

Let $\Im$ be a proper closed Lie ideal of $B$ such that $\Im \supset P$. The hypothesis (7.2) implies that $\Im^{L} \neq(0)$. Corollary 3.3 and its proof imply that each minimal dual ideal of $B$ is of the form $\Re(Q)$ for $Q$ a primitive ideal of $B$. If $\Im^{L} \cap \Re(Q)=(0)$ for all primitive ideals $Q$, then $\left[\mathfrak{S}^{L}, \mathfrak{R}(Q)\right] \subset \mathfrak{\Im}^{L} \cap \Re(Q)=(0)$, so by Corollary $3.3, \quad\left[\mathfrak{\Im}^{L}, B\right]=(0)$ and $\Im^{L} \subset Z=(0)$ which is a contradiction. Thus there exists a primitive ideal $Q_{0}$ such that $\mathfrak{\Im}^{L} \cap \Re\left(Q_{0}\right) \neq(0)$. Lemma 7.11 then yields $\left[\Re\left(Q_{0}\right), \Re\left(Q_{0}\right)\right] \subset \mathfrak{\Im}^{L}$. Lemma 7.12 then implies that $\mathfrak{\Im} \subset \mathfrak{\Im}^{L L} \subset\left[\Re\left(Q_{0}\right)\right.$, $\left.\Re\left(Q_{0}\right)\right]^{L}=Q_{0}$. As noted in the proof of Theorem 3.2, the resulting inequality $Q_{0} \subset P$ with $Q_{0}$ and $P$ primitive ideals implies that $Q_{0}=P$ and consequently $\mathfrak{\Im}=P$.

7.14. Lemma. Each minimal closed Lie ideal of $B$ is of the form $[\Re(Q), \Re(Q)]$ for some primitive ideal $Q$ of $B$.

Let $(D$ be a minimal closed Lie ideal of $B$. As noted in the proof of Lemma 7.13, $\mathscr{D} \cap \Re(Q) \neq(0)$ for some primitive ideal $Q$ of $B$; and so $\mathfrak{D} \subset \Re(Q)$. Thus by Lemma $7.11,[\Re(Q), \mathfrak{R}(Q)]=\mathfrak{D}$ by the minimal nature of $\mathfrak{D}$.

7.15. THEOREM. If $B$ is a semi-simple Banach algebra which satisfies (7.2) and (7.3), then $B$ is the direct topological sum of its minimal closed Lie ideals.

In view of Lemma 7.14, and the fact that any two ideals $\Re(Q)$, with $Q$ primitive, have only zero in common, a sum of minimal closed Lie ideals is a direct sum. Let $\Im$ be the direct topological sum of the minimal closed Lie ideal in $B$. If $\mathfrak{F} \neq B$, then (7.2) implies $\mathfrak{\Im}^{L} \neq(0)$. The argument used in the proof of Lemmas 7.13 and 7.14 shows that there is a primitive ideal $Q$ such that $\Im^{L} \cap \Re(Q) \neq(0)$. Lemma 7.11 then implies that $[\Re(Q), \Re(Q)] \subset \mathfrak{\Im}^{L}$. But then $[\Re(Q), \mathfrak{R}(Q)] \subset \mathfrak{\Im}^{L} \cap \mathfrak{F}=$ $(0)$, which is a contradiction.

In the final portion of this section, $B$ will denote a simple Banach algebra with an identity $u$, which satisfied (7.2) and

(7.4) The center 3 of $B$ consists of the complex multiples of the identity $u$ of $B$. 
In view of the proof of Theorem 7.5, we note that (7.1) is automatically satisfied by $B$. Thus if $\mathfrak{L}(I)=(0)$, for an ideal $I$, either $I$ is dense in $B$ or $I$ is a maximal ideal of deficiency one in $B$.

7.16. Lemma. If $\Re(M) \neq(0)$ for each maximal ideal $M$ of $B$, then each primitive ideal of $B$ is a maximal ideal. Furthermore, $B$ is the direct topological sum of its minimal dual ideals.

Since $B$ has an identity, each primitive ideal $P$ is contained in a maximal ideal $M$. If $M \neq P$, then by the argument used in the proof of Theorem 3.2, $\Re(M)=(0)$, which is a contradiction. Thus each primitive ideal is a maximal ideal. If $B$ is a primitive algebra, then $B$ is a simple algebra and thus is the only minimal dual ideal. Otherwise let $J$ be the direct topological sum of the minimal dual ideals $\Re(P)$, where $P$ is a primitive ideal. If $J$ were proper, there would exist a maximal ideal $N$ such that $N \supset J \supset \Re(N) \neq(0)$, which would contradict the semi-simplicity of $B$. Thus $J=B$.

7.17. Lemma. If $M$ is a maximal ideal of $B$ with $\Re(M)=(0)$, then $B=M \oplus K u$, where $K$ is the complex field. Moreover, $M$ as an algebra satisfies condition (7.2) and (7.3).

By Lemma 7.3 $M$ has deficiency one and thus $B=M \oplus K u$. Since $M$ is a maximal ideal of $B, M$ is a semi-simple Banach algebra and the center of $M$ is contained in the center of $B$, so is zero. If $\mathfrak{U}$ is a proper closed Lie ideal of $M$, and $\mathfrak{U}$ contains $V=\{x \in M:[x, y]=0$, $\forall y \in \mathfrak{U}\}$, then $\mathfrak{u}+K u$ is a proper closed Lie ideal of $B$ and $(\mathfrak{u}+K u)^{L}=$ $\mathfrak{H}^{L}=V+K u$, so $(\mathfrak{U}+K u) \supset(\mathfrak{U}+K u)^{L}$. The hypothesis (7.2) yields $\mathfrak{U}+K u=B$, which is a contradiction. Thus $M$ as an algebra satisfies (7.2).

7.18. THeOREM. Let $B$ be a simple Banach algebra with satisfies (7.2) and (7.4). The only nonzero closed Lie ideals of $B$ are either $B$ and $K u$ or are $B, K u$ and $[B, B]$, in which case $B=[B, B] \oplus$ $K u$, and $B$ is the direct sum of the minimal closed Lie ideals of $B$.

Since $B$ is simple, by a theorem of Herstein [3, p. 282], any closed Lie ideal is either contained in $K u$ or else contains $[B, B]=\mathfrak{\Omega}$.

Suppose first that $K u \subset \mathbb{Q}$. From Herstein's theorem applied to $\mathfrak{Q}^{L}$, either (a) $\mathbb{2}^{L} \subset K u \subset \mathbb{Q}$ or (b) $\mathfrak{Q} \subset \mathbb{2}^{L}$. In case (a), the relation (7.2) yields $[B, B]=B$, so that the only closed Lie ideals are $K u$ and $B$. In case (b) $\mathfrak{2}^{L} \supset \mathbb{2}^{L L}$ so that, by (7.2), $\mathfrak{2}^{L}=B$. Thus $2 \subset 3$. In view of the reasoning for Theorem $6.2,[B, B]=(0)$. Thus $B$ is commutative and 
so $B=K u$. Hence the case $K u \subset \mathbb{Q}$ leads to the first possibility stated in the theorem.

If $K u \not \subset[B, B]$, let $\mathfrak{I}=[B, B] \oplus K u$. Suppose $\mathfrak{I} \neq B$. Another application of Herstein's theorem yields $\mathfrak{I}^{L} \supset[B, B]$ or $\mathfrak{T}^{L} \subset K u \subset \mathfrak{T}$. The latter is in contradiction to (7.2), while the former implies $\mathfrak{I}^{L} \supset \mathfrak{I}$ so $\mathfrak{I}^{L L} \subset \mathfrak{I}^{L}$. Thus $\mathfrak{T}^{L}=B$, which as above shows that $B$ is the complex field. Hence $B=[B, B]+K u$, in which case $B$ is the direct sum of the minimal closed Lie ideals of $B$.

7.19. THEOREM. Let $B$ be a semi-simple Banach algebra which satisfies (7.2) and (7.4). Suppose that $B$ is not simple, and is not commutative. Then $B$ is the direct topological sum of its minimal closed Lie ideals and is either the direct topological sum of its minimal dual ideals, or the minimal dual ideals and the center.

Suppose that $\Re(M) \neq(0)$ for each maximal ideal $M$ of $B$. Then by Lemma 7.16 each primitive ideal of $B$ is a maximal ideal and $B$ is the direct topological sum of its minimal dual ideals. Also, as noted in the proof of Lemma 7.16, each minimal dual ideal has the form $\Re(M)$ for a maximal ideal.

Let $M$ be any maximal ideal of $B$, and let $\mathfrak{F} \neq(0)$ be a closed Lie ideal, $\mathfrak{\Im} \subset \Re(M)$. Then, by a result of Herstein [3, p. 281], either $S(\mathfrak{\Im})$ is in the center of $B$ or there is a nonzero ideal $I$ in $S(\Im)$, in which case we may assume $I$ is the largest such ideal. The first possibility

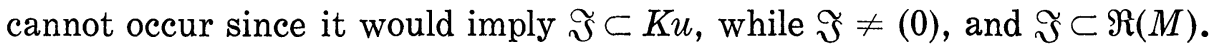
Thus the second possibility holds. If $a \in I \cap M$, then for all $x \in B$, $[a, x] \in \mathfrak{\Im} \subset \Re(M)$, while $[a, x] \in M$, so $[a, x]=0$ for all $x \in B$. Thus $a$ is central and since $u \notin M$, we conclude $a=0$. Consequently $I \cap M=(0)$ and $B=M \oplus I$ so that $I=\Re(M)$. By a result of Zuev [14],

$$
\mathfrak{\Im} \supset[I, B] \supset[\Re(M), \mathfrak{R}(M)] \text {. }
$$

Consequently each Lie ideal $[R(M), R(M)]$ is a minimal closed Lie ideal of $B$. Let $\mathfrak{u}$ be the direct topological sum of its minimal closed Lie ideals of $B$. Since $B$ is the direct topological sum of the minimal dual ideals and these have the form $\mathfrak{R}(M)$, we see that $\mathfrak{U} \supset[B, B]+K u$. Another application of the result of Herstein used above shows that either $S\left(\mathfrak{U}^{L}\right) \subset K u$ or these exists a nonzero ideal $N$ such that $N \subset S\left(\mathfrak{U}^{L}\right)$. The first possibility implies that $\mathfrak{U}^{L}=K u$, whence $\mathfrak{U} \supset \mathfrak{U}^{L}$ so by (7.2) $\mathfrak{U}=B$. In the second instance, let $a \neq 0, a \in N$. Then for all $x \in B$, $[a, x] \in \mathfrak{U}^{L} \cap \mathfrak{U}=\mathfrak{W}$, from which it is immediate that $\mathfrak{W}^{L L} \subset \mathfrak{B}^{L}$. Thus by (7.2) $\mathfrak{W}^{L}=B$, so $\mathfrak{W} \subset K u$, and by the argument used in Theorem 6.2 we deduce that $a \in K u$, which implies that $N=S\left(\mathfrak{u}^{L}\right)=B$. We therefore conclude that $[B, B] \subset \mathfrak{U} \cap \mathfrak{U}^{L} \subset K u$, so as argued earlier $B$ 
is commutative, which is a contradiction.

In the event that $\Re(M)=(0)$ for some maximal ideal $M$ of $B$, it follows from Lemma 7.17 that $B=M \oplus K u$ where $K$ is the complex field, and $M$ as an algebra satisfies (7.2) and (7.3). Then by Theorem 7.15 $M$ is the direct topological sum of its minimal closed Lie ideals and by Corollary 3.3 is the direct topological sum of the minimal dual ideals of $M_{0}$. Any minimal closed Lie ideal of $M$ is one of $B$, and any minimal dual ideal is one of $B$. Since $K u$ is a minimal closed Lie ideal of $B$, the theorem follows.

\section{REFERENCES}

1. R. Arens and I. Kaplansky, Topological representation of algebras, Trans. Amer. Math. Soc. 63 (1948), 457-481.

2. F. Bonsall and A. W. Goldie, Annihilator algebras, Proc. London Math. Soc. (3) 4 (1954), 154-167.

3. I. N. Herstein, On the Lie and Jordan rings of a simple, associative ring, Amer. J. Math. 77 (1955), 279-285.

4. -__ Jordan homomorphisms, Trans. Amer. Math. Soc. 81 (1956), 331-341.

5. __, Lie and Jordan structures in simple, associative rings, Bull. Amer. Math. Soc. 67 (1961), 517-531.

6. N. Jacobson, Structure of rings, Amer. Math. Soc. Colloq. Publ. vol. 37, 1956.

7. ___ Lie algebras, New York (1962).

8. R. V. Kadison, Isometries of operator algebras, Ann. of Math. 56 (1952,, 494-503.

9. I. Kaplansky, Semi-automorphisms of rings, Duke Math. J. 14 (1947), 521-527.

10. C. E. Rickart, General theory of Banach algebras, Princeton (1960).

11. B. Yood, Topological properties of homomorphisms between Banach algebras, Amer, J. Math. 76 (1954), 155-167.

12. - Homomorphisms on normed algebras, Pacific J. Math. 8 (1958), 373-381.

13. ____ Faithful *-representations of normed algebras, Pacific J. Math. 10 (1960), 345-363.

14. I. I. Zuev, Lie ideals of associative rings, (Russian) Uspehi Mat. Nauk 18 (1963), no. 1 (109), 115-158.

15. E. Størmer, Positive linear maps on operator algebras, Acta. Math. 110 (1963), 233-278.

UNIVERSITY OF OREGON 



\section{PACIFIC JOURNAL OF MATHEMATICS}

\section{EDITORS}

\author{
H. Samelson \\ Stanford University \\ Stanford, California \\ R. M. Blumenthal \\ University of Washington \\ Seattle, Washington 98105
}

\author{
J. Dugundu \\ University of Southern California \\ Los Angeles, California 90007 \\ *Richard Arens \\ University of California \\ Los Angeles, California 90024
}

\section{ASSOCIATE EDITORS}
E. F. BeCKENBACH
B. H. NeUMANN
F. WOLF
K. YosidA

\section{SUPPORTING INSTITUTIONS}

\author{
UNIVERSITY OF BRITISH COLUMBIA \\ CALIFORNIA INSTITUTE OF TECHNOLOGY \\ UNIVERSITY OF CALIFORNIA \\ MONTANA STATE UNIVERSITY \\ UNIVERSITY OF NEVADA \\ NEW MEXICO STATE UNIVERSITY \\ OREGON STATE UNIVERSITY \\ UNIVERSITY OF OREGON \\ OSAKA UNIVERSITY \\ UNIVERSITY OF SOUTHERN CALIFORNIA
}

\author{
STANFORD UNIVERSITY \\ UNIVERSITY OF TOKYO \\ UNIVERSITY OF UTAH \\ WASHINGTON STATE UNIVERSITY \\ UNIVERSITY OF WASHINGTON \\ * * * * \\ AMERICAN MATHEMATICAL SOCIETY \\ CALIFORNIA RESEARCH CORPORATION \\ SPACE TECHNOLOGY LABORATORIES \\ NAVAL ORDNANCE TEST STATION
}

Mathematical papers intended for publication in the Pacific Journal of Mathematics should by typewritten (double spaced). The first paragraph or two must be capable of being used separately as a synopsis of the entire paper. It should not contain references to the bibliography. No separate author's resumé is required. Manuscripts may be sent to any one of the four editors. All other communications to the editors should be addressed to the managing editor, Richard Arens, at the University of California, Los Angeles, California 90024.

50 reprints per author of each article are furnished free of charge; additional copies may be obtained at cost in multiples of 50 .

The Pacific Journal of Mathematics is published quarterly, in March, June, September, and December. Effective with Volume 13 the price per volume (4 numbers) is $\$ 18.00$; single issues, $\$ 5.00$. Special price for current issues to individual faculty members of supporting institutions and to individual members of the American Mathematical Society: $\$ 8.00$ per volume; single issues $\$ 2.50$. Back numbers are available.

Subscriptions, orders for back numbers, and changes of address should be sent to Pacific Journal of Mathematics, 103 Highland Boulevard, Berkeley 8, California.

Printed at Kokusai Bunken Insatsusha (International Academic Printing Co., Ltd.), No. 6, 2-chome, Fujimi-cho, Chiyoda-ku, Tokyo, Japan.

PUBLISHED BY PACIFIC JOURNAL OF MATHEMATICS, A NON-PROFIT CORPORATION

The Supporting Institutions listed above contribute to the cost of publication of this Journal, but they are not owners or publishers and have no responsibility for its content or policies.

* Basil Gordon, Acting Managing Editor until February 1, 1966. 


\section{Pacific Journal of Mathematics}

\section{Vol. 15, No. $3 \quad$ November, 1965}

David R. Arterburn and Robert James Whitley, Projections in the space of

bounded linear operators .................................

Robert McCallum Blumenthal, Joram Lindenstrauss and Robert Ralph Phelps,

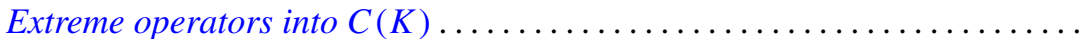

L. Carlitz, A note on multiple exponential sums ................... 757

Joseph A. Cima, A nonnormal Blaschke-quotient .................... 767

Paul Civin and Bertram Yood, Lie and Jordan structures in Banach algebras . . . 775

Luther Elic Claborn, Dedekind domains: Overrings and semi-prime

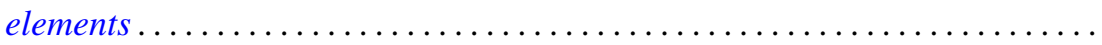

799

Luther Elic Claborn, Note generalizing a result of Samuel's .............. 805

George Bernard Dantzig, E. Eisenberg and Richard Warren Cottle, Symmetric

dual nonlinear programs ................................... 809

Philip J. Davis, Simple quadratures in the complex plane ............... 813

Edward Richard Fadell, On a coincidence theorem of F. B. Fuller ............ 825

Delbert Ray Fulkerson and Oliver Gross, Incidence matrices and interval

graphs ........................................ 835

Larry Charles Grove, Tensor products over $H^{*}$-algebras ..................

Deborah Tepper Haimo, $L^{2}$ expansions in terms of generalized heat polynomials

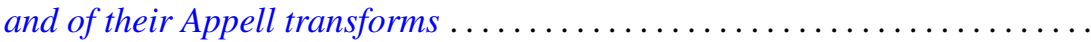

I. Martin (Irving) Isaacs and Donald Steven Passman, A chardcterization of groups in terms of the degrees of their characters ..........

Donald Gordon James, Integral invariants for vectors over local fields........ 905

Fred Krakowski, A remark on the lemma of Gauss ................... 917

Marvin David Marcus and H. Minc, A subdeterminant inequality ........... 921

Kevin Mor McCrimmon, Norms and noncommutative Jordan algebras ........ 925

Donald Earl Myers, Topologies for Laplace transform spaces ............... 957

Olav Njstad, On some classes of nearly open sets .................... 961

Milton Philip Olson, A characterization of conditional probability ........... 971

Barbara Osofsky, A counter-example to a lemma of Skornjakov .............. 985

Sidney Charles Port, Ratio limit theorems for Markov chains ............... 989

George A. Reid, A generalisation of $W^{*}$-algebras ...................... 1019

Robert Wells Ritchie, Classes of recursive functions based on Ackermann's

function ........................................... 1027

Thomas Lawrence Sherman, Properties of solutions of $n$th order linear

differential equations ........................................ 1045

Ernst Snapper, Inflation and deflation for all dimensions . .............. 1061

Kondagunta Sundaresan, On the strict and uniform convexity of certain Banach

spaces............................................. 1083

Frank J. Wagner, Maximal convex filters in a locally convex space .......... 1087

Joseph Albert Wolf, Translation-invariant function algebras on compact 